\title{
L'actionnariat Salarié Et La Création De Valeur Partenariale Dans Les Entreprises Au Cameroun
}

\author{
Ousmanou Alim, \\ $\mathrm{PhD}$ en Finance Université de Buea, Cameroun \\ Tsapi Victor, \\ Professeur en Sciences de Gestion Université de Ngaoundéré, Cameroun
}

Doi:10.19044/esj.2019.v15n34p250 URL:http://dx.doi.org/10.19044/esj.2019.v15n34p250

\section{Résumé}

L'objet de ce travail est d'examiner l'impact de l'actionnariat salarié sur la création de valeur partenariale dans les entreprises au Cameroun. L'échantillon est constitué de 89 entreprises dont 31 disposent la politique d'actionnariat salarié. Les données utilisées sont de source secondaire issue de l'Institut National de la Statistique du Cameroun. Le niveau moyen de participation des salariés au capital se situe à hauteur de 7,4\%. L'analyse de régression multiple en données de panel conclut que la participation des salariés au capital affecte positivement et significativement la valeur partenariale et la part de la valeur partenariale distribuée aux salariés. Cette recherche consacre les postulats de la théorie des parties prenantes et montre que l'actionnariat salarié est un levier de création de richesse partenariale dans les entreprises au Cameroun.

Mots clés : Actionnariat salarié, Création de valeur partenariale, Théorie des parties 


\title{
Employee Ownership and Creating Stakeholder Value of compagnies in Cameroon
}

\author{
Ousmanou Alim, \\ PhD en Finance Université de Buea, Cameroun \\ Tsapi Victor, \\ Professeur en Sciences de Gestion Université de Ngaoundéré, Cameroun
}

\begin{abstract}
The purpose of this work is to examine the impact of employee ownership on the creation of stakeholder's value in companies in Cameroon. The sample includes 89 companies, of which 31 practice employee ownership policy for at least 10 years. Data used come from secondary source, collected over the 2008-2013 period at the National of Statistical Institute of Cameroon. These data were analyzed through a multiple regression in panel data, corrected for any latent heteroskedasticity and serial auto-correlation. The paper finds that, the average level of participation of employee in the capital is $7.4 \%$. For the results of model estimates, the percentage of capital held by employees affect positively and significantly the creation of stakeholder's value in companies in Cameroon. This research dedicates the assumptions of the stakeholder theory and shows that employee ownership is a lever for creating wealth for all in companies in Cameroon.
\end{abstract}

Keywords: Employee Ownership, Creation of stakeholder's value, Stakeholder theory

\section{Introduction}

Depuis la fin des années 1980, l'actionnariat salarié fait l'objet d'une attention croissante dans le cadre de la gouvernance d'entreprise. En effet, l'évolution du système de gouvernance a fait naître une nouvelle forme de participation dans les entreprises. Les salariés peuvent constituer un portefeuille de valeur mobilière et acquérir souvent, dans des conditions avantageuses des actions de la société qui les emploie, avec pour effet supposé de stimuler l'effort collectif et d'obtenir un accroissement de la valeur des entreprises (Dondi, 1992 ; Desbrières, 2002 et Autenne, 2005). Selon l'Observatoire de l'actionnariat salarié en Europe, une enquête menée auprès de quelques analystes financiers européens confirme non seulement l'intense 
intérêt pour le sujet, mais également leur volonté d'être mieux informés en la matière.

La littérature sur l'actionnariat salarié a tout d'abord cherché à étudier ses liens avec la performance organisationnelle. Les résultats de ces études se sont avérés mitigés, bien qu'ils aboutissent globalement soit à des effets positifs, soit à des effets négligeables (Buchko, 1993 ; Gamble et al. 2002 ; Trewhitt, 2000). Par ailleurs, la majorité des études prônant les bienfaits de l'actionnariat salarié ont souvent appuyé leur position en soulignant ses effets sur les et attitudes des salariés (Long, 1980 ; Gamble et al.,2002 Caramelli, 2006), ainsi que sur la productivité (Collat, 1988 ; Ben-Ner et Jones, 1995 ; Jones et Jato, 1995) tout en négligeant son impact sur la création de valeur. En outre, les travaux empiriques disponibles (Collat, 1988 ; Chang et Mayers, 1992 ; Dondi, 1992 ; Trébucq, 2002 ; Poualin-Rehm, 2007 ; Guédri et Hollandts, 2008 a) étudiant la relation entre actionnariat salarié et création de valeur, présentent des résultats peu significatifs, du fait de leurs conclusions contradictoires. De surcroît, la totalité de ces études ont traité de la création de valeur actionnariale, à l'exception, à notre connaissance, de quelques travaux (Poulain-Rehm, 2007 et Garffata, 2011) qui ont eu le mérite d'aborder la création de valeur sous un angle à la fois actionnarial et partenarial.

Ce papier contribue au débat actuel pour examiner l'impact de l'actionnariat salarié sur la création de valeur partenariale. Deux questionnements guident cette problématique : quel est le niveau de participation des salariés au capital de leur entreprise? Peut-il être un facteur de création et de redistribution de valeur partenariale ?

Le choix de cette problématique se justifie par les explications suivantes. D'abord, il n'est pas facile de trouver des évidences sur ce thème dans la littérature africaine en générale et camerounaise en particulier. La seule étude menée dans le contexte camerounais (à notre connaissance) montre d'une part, un lien positif et significatif entre l'actionnariat salarié et la valeur ajoutée et, d'autre part, une relation négative entre l'actionnariat salarié et la productivité (Ngongang, 2013). Cette étude, menée dans un contexte où le niveau moyen de participation des salariés au capital était de l'ordre de 2,9\%, n'inclut pas les exigences liées au développement d'un actionnariat salarié significatif (un niveau moyen de participation des salariés au capital de l'ordre de $3 \%$ au moins).

Ensuite, la quasi-totalité des études menées sur ce sujet ont été réalisées dans le contexte où les marchés financiers sont efficients. Enfin, l'étude du lien entre actionnariat salarié et création de valeur semble aujourd'hui dépourvue de tout intérêt, compte tenu du nombre des travaux réalisés dans ce sens. Toutefois, il semble raisonnable de se repencher sur cette question, particulièrement après la crise financière de 2008 qui a secoué le monde entier. En effet, les enquêtes menées auprès des entreprises à 
actionnariat salarié au cours des dernières années ont révélé que ces firmes ont résisté à la crise. C'est dans ce sens qu'une étude menée par la fédération des actionnaires salariés en Europe en 2017 a montré que : 70\% des entreprises américaines à actionnariat salarié (contre $65 \%$ en 2016) ont enregistré une meilleure performance économique que l'année précédente, $78 \%$ ont vu leur chiffre d'affaire augmenté par rapport à 2016 et près de $70 \%$ (contre $64 \%$ en 2016) ont obtenu des meilleurs rendements financiers.

Ce papier est structuré comme suit. La première section présente les principaux développements théoriques portant sur la relation entre l'actionnariat salarié et la création de valeur partenariale. La deuxième section décrit la méthodologie utilisée pour tester les hypothèses et les principaux résultats obtenus, avant de procéder à leurs analyses, tandis que la troisième présente la conclusion et les recommandations.

\section{Revue de la littérature}

Dans le cadre de l'approche traditionnelle de la gouvernance, l'actionnariat salarié se justifie par la relation d'agence, dans son acceptation classique, entre les salariés et les dirigeants. En effet, séparer la fonction de décision (déléguée aux dirigeants par les actionnaires) de la fonction de mise en œuvre (confiée aux salariés) est une illustration de la relation d'agence liant le principal (ou mandant) avec l'agent (ou mandataire). Dans cette illustration, le principal (le dirigeant) veut de l'agent (le salarié) qu'il agisse au mieux de ses intérêts, ce qui implique une délégation de pouvoir en contrepartie d'une rémunération. De sa part, ce dernier s'engage à agir conformément aux intérêts du principal.

Toutefois, une relation d'agence devient conflictuelle en cas de divergence d'intérêt entre le principal et l'agent, et d'imparfaite observabilité des coûts d'établissement et d'exécution des contrats. Les conflits opposants les dirigeants et salariés proviennent fondamentalement du caractère incomplet des contrats (Caby et Hrigoyen, 2001 ; Desbrières, 2002) et de la tendance que chacun a à privilégier son propre intérêt. Notamment, les salariés peuvent adopter des comportements allant à l'encontre des intérêts des dirigeants et actionnaires. Par exemple la réduction des efforts individuels afin de compenser le caractère non diversifiable de leur investissement en capital humain (Desbrières, 2002).

Pour donc limiter les sources de conflits et assurer la convergence des intérêts des salariés avec ceux des dirigeants et actionnaires, des mécanismes d'incitation et de contrôle doivent être mise en place comme l'actionnariat salarié. Dans ce cadre Long (1980) ) estime que l'actionnariat salarié pourrait réduire les coûts d'agence en alignant les intérêts des salariés à ceux des actionnaires. L'idée est que la détention d'actions par les salariés aligne leurs intérêts à ceux des actionnaires externes et constitue par conséquent, un 
mécanisme d'incitation contribuant à réaliser l'objectif de la firme à savoir, la maximisation de la valeur des fonds propres ou la valeur actionnariale (Desbrières, 2002). Aussi, cette détention peut conférer aux salariés certains droits de contrôle pouvant servir à renforcer le gouvernement d'entreprise dans sa dimension actionnariale. Dans ce sens, Bompoint et Marois (2004, p.56) précisent que « l'actionnariat salarié est l'émergence d'un nouvel acteur financier. Le salarié actionnaire devient un partenaire qui investit dans son entreprise...il contribue à renforcer la gouvernance d'entreprise ».

Considérée comme une approche trop restrictive, la théorie traditionnelle de la gouvernance a été contestée par les auteurs appartenant au courant qu'on peut qualifier de « stakeholder capitalism ». En effet, de nombreux auteurs (Freeman, 1984 ; Garvey et Swan, 1994 ; Clarkson, 1995 ; Charreaux, 1997a ; Hirigoyen, 1997 ; Stout, 2007) ont adopté une vision plurale de la firme où les intérêts de l'ensemble de ses partenaires sont réellement pris en considération; et où encore la notion de créancier résiduel n'est plus un attribut exclusif des actionnaires, mais peut également s'appliquer à des partenaires tels que les salariés (Blair, 1995).

La reconnaissance du rôle important des différents partenaires, et en particulier les salariés a conduit à une remise en cause de la vision actionnariale dans les firmes, au profit d'une conception partenariale qui vise à assurer un équilibre entre toutes les parties prenantes. Dans cet esprit Hill et Jones (1992) avancent une théorie de l'agence élargie ou généralisée où tous les partenaires sont explicitement pris en compte ; tandis que Laffont et Martimort (1997) considèrent la firme comme étant un ensemble des contrats multilatéraux entre chaque stakeholders (principal) et le dirigeant (agent commun). Dans le cadre de cette nouvelle approche de gouvernance, le positionnement de l'actionnariat salarié se justifie par la relation d'agence entre salariés et dirigeants. Cependant, cette relation d'agence se présente avec une seconde acceptation, très différente de la première déjà présentée au niveau de l'approche traditionnelle, dans laquelle on peut voir les salariés louer leur capital humain spécifique (qui est de nature risquée) au dirigeant (Blair, 1997), et perçoivent en contrepartie une quasi-rente (Rottenberg, 1962).

Le schéma conceptuel traditionnel de la relation d'agence est donc inversé : le salarié n'est plus considéré comme l'agent mais le principal de la relation contractuelle qu'il entretient avec la firme. Toutefois et du fais toujours de l'incomplétude des contrats, des conflits opposants salariés et dirigeants peuvent émerger de cette relation contractuelle. En effet, les salariés courent des risques au titre de leur capital humain spécifique. Blair (1997) présente de manière précise ces risques et en distingue deux types : le risque que la rente ou les quasi-rentes générées par le capital humain spécifique soient expropriées ex post par les autres parties prenantes (principalement les 
dirigeants et les actionnaires) et le risque que la valeur actuelle du capital humain spécifique fluctue à l'avenir, soit parce que les compétences ne seraient plus utiles à la firme, soit parce que la firme ne générait plus autant de rentes.

Si d'une manière ou d'une autre, ce risque n'est pas réduit ou rémunéré, l'incitation à développer le capital humain spécifique disparaît, ce qui est nuisible et dommageable à l'ensemble des parties prenantes. En effet, l'importance du capital humain est de plus en plus soulignée par les chercheurs et les praticiens comme étant la caractéristique essentielle des entreprises du 21ème siècle (Garfatta, 2010). Une partie de la valeur créée par l'entreprise fait appel certes au capital intellectuel, social et culturel possédé par les salariés. Face à cette importance accrue du capital humain spécifique dans la vie organisationnelle et vu l'impossibilité de supprimer le risque encouru par les salariés, sa rémunération semble être une exigence. Dans ce sens Blair (1997) propose de rémunérer ce risque via l'actionnariat salarié. Selon cet auteur "la rémunération des employés par des actions des entreprises peut fournir un mécanisme destiné à encourager et à protéger les investissements en capital humain spécifique. La détention d'actions par les salariés fonctionne comme une sorte d'otage permettant de crédibiliser la promesse des firmes de partager les rentes- (...), elle donne aussi certains droits de contrôle et simultanément aligne les intérêts des employés et des détenteurs externes d'actions ».

L'idée est que la détention d'actions par le salarié lui confère certains droits de contrôle qui peut exercer, en tant que principal dans sa relation d'agence, sur le dirigeant, agent de la même relation. Un tel contrôle protège les investissements en capital humain spécifique et par, conséquence, encourage leur développement. De surcroît, cette détention permet l'alignement des intérêts des salariés et des actionnaires externes, ce qui peut favoriser l'instauration d'un régime de confiance dans l'organisation (Charreaux, 1998; Poulain-Rehm, 2007 et le développement d'une coopération entre les parties prenantes (Aoki, 1984; Marens et Alii, 1999). De telles conséquences contribuent certes à la réalisation de l'objectif de la firme dans sa nouvelle conception partenariale, à savoir, maximiser la création de la valeur partenariale (stakeholders value) tout en assurant l'équilibre entre les différentes parties prenantes (Fama, 1980 ; Blair, 1996 ; Hirigoyen, 1997).

Bien que sur le plan théorique, son positionnement est pleinement justifié dans la double perspective de la gouvernance actionnariale et partenariale ; et que sur le plan pratique, il est en développement progressif, le thème actionnariat salarié et la création de valeur partenariale reste l'objet d'un ensemble des travaux très peu étendu. Ainsi, l'on note quelques travaux empiriques réalisés dans ce sens. A partir d'un échantillon de 131 sociétés françaises cotées sur l'indice SBF 120, dont 24 à actionnariat salarié significatif, Poulain-Rehm 
(2007) trouve une absence d'effet de l'actionnariat salarié sur la création de valeur partenariale (mesurée par la répartition de la valeur ajoutée allouée aux salariés). Toutefois, dans le même contexte et se basant sur les données de panel relatives à 121 entreprises observées sur une période d'analyse de 5 ans (2004-2008), Garfatta (2010) souligne que l'actionnariat salarié présente un effet positif et significatif au seuil de $1 \%$ sur la création de valeur partenariale (mesurée par la valeur ajoutée). Dans le contexte camerounais, Ngongang (2013) à travers un échantillon de 64 entreprises dont 32 disposent de la politique d'actionnariat salarié, montre que l'actionnariat salarié influence positivement et significativement la valeur ajoutée. Ainsi, au regard des analyses qui précèdent, nous pouvons émettre l'hypothèse suivante : Hypothèse 1 : la participation des salariés au capital de leur entreprise impacte positivement sur la valeur partenariale.

Rappelons que l'objectif de la firme dans sa nouvelle conception partenariale est de maximiser la création de valeur partenariale tout en assurant l'équilibre entre les différentes parties prenantes (Fama, 1980 ; Blair, 1996 ; Charreaux et Desbrières, 1998). Donc, la problématique de la valeur partenariale soulève immanquablement celle des destinataires de la valeur créée et, par conséquence, de sa répartition (Poulain-Rehm, 2007). A cet égard et étant donné que le rôle de l'actionnariat salarié est de défendre et protéger les intérêts des employés, nous émettons une deuxième hypothèse qui se formule comme suit :

Hypothèse 2 : la participation des salariés au capital de leur entreprise a un effet positif sur la part de valeur partenariale allouée aux salariés.

\section{Méthodologie}

\section{Echantillon et source des données}

Cette étude porte sur 89 entreprises issues de la base de données de l'Institut National de la Statistique du Cameroun. De ces 89 entreprises, 31 disposent une politique d'actionnariat salarié. Afin de les identifier, la méthode d'échantillonnage par convenance a été utilisée. Pour ce faire, l'étude s'est appuyée principalement sur les entreprises publiques et parapubliques privatisées dans les années 1990 ; dans la mesure où l'ordonnance N90/004 du 22 juin 1990 et le décret N90/1257 du 30 Août 1990 portant application de l'ordonnance ci-dessus; consacrent l'actionnariat salarié dans les entreprises privatisées à hauteur de 3 à $5 \%$ du capital ${ }^{26}$. Au terme d'un recensement des dites entreprises, il a été enregistré 24 entreprises privatisées dont 03 ont été exclues sur la base des critères suivants :

Les sociétés n'ayant pas rétrocédé la part de $5 \%$ du capital appartenant aux salariés ;

\footnotetext{
${ }^{26}$ Bulletin du GICAM, 32, 2006.
} 
- Les sociétés dont les salariés ont renoncé à la politique d'actionnariat salarié ;

- $\quad$ Les banques, établissement de crédits et sociétés immobilières compte tenu de leurs caractéristiques financières spécifiques.

Par la suite, les annonces du programme d'actionnariat salarié dans les filiales des sociétés étrangères au Cameroun, comme le « Share plan 2013 » d'AxaAssurance ou celui de SANOFI ont permis de recenser 10 autres entreprises à actionnariat salarié ${ }^{27}$.

Pour les 58 sociétés conventionnelles, elles ont été choisies sur la base d'un seul critère: la performance. En effet, chaque année, l'Institut national des statistiques du Cameroun classe les 100 premières entreprises du Cameroun en termes de performance financière (chiffre d'affaires, bénéfices). Ainsi, les 58 sociétés conventionnelles sélectionnées dans l'échantillon font partie de cette classification, ainsi que les 31 sociétés disposant la politique d'actionnariat salarié.

Les données collectées sont de source secondaire. Elles proviennent de la base de données de l'INS ${ }^{28}$ (Institut National de la Statistique). Dans cette base, l'on retrouve des bilans et des comptes du résultat des entreprises. Ces différents documents sont accessibles après autorisation du Directeur Général de l'INS. Les données (comptables et financières) extraites de cette base de données, couvrent la période $2008-2013^{29}$, soit 6 ans.

\section{Définition et mesures des variables}

La variable dépendante ici est la création de valeur partenariale tans dis que la variable indépendante est l'actionnariat salarié. La création de valeur partenariale est mesurée par la valeur partenariale et la part de valeur partenariale allouée aux salariés. Dans ce sens, il faut signaler que la mesure de la valeur partenariale reste jusqu'à nos jours un puzzle à résoudre par conséquent une piste de recherche qui nécessite beaucoup de réflexions.

Charreaux et Desbrières (1998) ont tenté d'apporter des éléments de réponse à cette problématique en proposant une méthode, qui s'appuie sur une mesure globale de la rente créée par la firme en relation avec les différents partenaires et non les seuls actionnaires. Cette dernière, pour séduisante qu'elle soit du point de vue conceptuel, si elle annonce incontestablement des voies de recherche prometteuses dans un proche avenir, souffre cependant, comme le reconnaissent les auteurs, d'un défaut d'opérationnalisation

\footnotetext{
${ }^{27}$ A ce niveau, nous avons suivi l'actualité de ces sociétés sur leur site internet au cours de la période 2011-2015.

${ }^{28}$ L'Institut National de la Statistique est un établissement public administratif créé par décret présidentiel $\mathrm{N}^{\circ} 2001 / 100$ du 20 avril 2001. Il est placé sous la tutelle technique du ministère en charge de l'économie. Il est doté d'une personnalité juridique et de l'autonomie financière. Son siège est fixé à Yaoundé, capitale politique du Cameroun.

${ }^{29} \mathrm{La}$ raison qui justifie le choix de cette période est le souci de travailler sur une période de reprise et de stabilité économique et monétaire du Cameroun : deux ans après l'atteinte du point d'achèvement PPTE et 6 ans après la crise de financière de 2008.
} 
puisqu'elle suppose l'identification des prix et des coûts d'opportunité pour les différentes parties prenantes.

Plus modestement, tout comme dans les travaux de Wanda (2001), Poulain-Rehm (2007), Garffata (2010) et Ngongang (2013) ; c'est la valeur ajoutée au sens comptable du terme, qui a été retenue comme mesure de valeur partenariale. De manière définitive, il a été retenu le logarithme neperien de la valeur ajoutée ${ }^{30}$. L'équation 1 ci-dessous donne la formule de cette valeur.

\section{Valeur partenariale $=$ Ln (Valeur ajoutée $)(1)$}

Par ailleurs, comme la problématique de la valeur soulève immanquablement celle des destinataires de la valeur créée, par conséquent, celle de sa répartition, l'on retient à la manière de Poulain-Rehm (2007) ou de Garfatta (2010), la répartition de la valeur ajoutée comme variable dépendante. Ainsi, elle peut se définir de la manière suivante :

\section{Part de valeur partenariale allouée auxsalariés = chargesdepersonne valeurajoutée

L'actionnariat salarié a été évalué en utilisant deux mesures. La première mesure a été définie par le pourcentage de capital détenu par les salariés et la seconde par les variables binaires $(1,0)$ relatives à la présence des salariés dans le capital de leurs entreprises. Cette opérationnalisation de l'actionnariat salarié est la plus utilisée dans la littérature sur la gouvernance d'entreprise (Trébucq, 2002; Poulain-Rehm, 2007; Guédri et Hollandts, 2008; Ngongang, 2013).

La création de valeur pouvant être influencée par des facteurs autres que l'actionnariat salarié, plusieurs variables de contrôle ont été incluses dans cette étude. Il a été contrôlé, en particulier, l'effet de la politique d'investissement, de la marge de l'entreprise, du niveau d'activité, de la taille de l'entreprise, du secteur d'activité, du temps et de privatisation.

Politique d'investissement: Les investissements ont été appréhendés à travers les dépenses en immobilisations déclarées en chiffre d'affaires (hors taxes) sur la période 2008-2013. Cette mesure a été adoptée par D'Arcimoles et Trebucq (2003) ou Ngongang (2013).

Marge de l'entreprise: Rappaport (1996) recommande de conserver la marge de l'entreprise en tant que variables clés de la création de richesse. Dans cette recherche, la marge brute d'exploitation est conservée et correspond au ratio entre le résultat brut d'exploitation et le chiffre d'affaires. Cette mesure a également été utilisée par Poulain-Rehm (2007) et Trebucq (2002).

\footnotetext{
${ }^{30} \mathrm{La}$ transformation logarithmique est communément utilisée afin d'obtenir une nouvelle variable ayant une distribution plus proche de celle de la loi normale.
} 
Le niveau d'activité: dans la mesure où Copeland et al. (1991) ont recommandé un niveau d'activité comme variable critique dans la création de richesse, la croissance du chiffre d'affaires (hors taxes) sur la période 20082013 a été introduite dans l'étude. Cette mesure a également été retenue par Trebucq (2002) et Ngongang (2013).

Taille de l'entreprise et secteur d'activité: la prise en compte des effets de taille et de secteur d'activité s'avère indispensable pour une vision comparative de la performance strictement financière des entreprises et, par extension, de la création de richesse. Une taille réduite peut en effet entraîner un plus grand contrôle des actionnaires sur les actions des gérants. La variable de taille a été opérationnalisée par le logarithme des actifs totaux (période 2008-2013). En outre, la création de richesse des entreprises doit être évaluée par rapport à la performance du secteur. Par conséquent, cette variable prenait en compte, sous la forme d'un regroupement des entreprises du sous-échantillon d'actionnariat salarié en deux catégories, celles appartenant au secteur industriel et celles appartenant aux autres secteurs (commerce, services, agriculture, etc.).

Effet temps: pour prendre en compte l'effet du temps passé par l'actionnariat salarié sur la création de richesse des entreprises, le nombre d'années pendant lequel la politique d'actionnariat salarié de l'entreprise a été incluse comme variable de contrôle dans le modèle. Ce choix est justifié par le fait que la littérature a montré qu'il faut du temps aux employés pour comprendre pleinement le mécanisme d'actionnariat des employés (Klein, 1987). Cela peut avoir un impact sur la manière dont les employés concevront ce mécanisme ainsi que sur leurs réactions comportementales (Caramelli, 2006).

Privatisation : la privatisation est incluse dans le modèle comme variable de contrôle pour deux raisons. Premièrement $67 \%$ des entreprises de l'échantillon sont des entreprises privatisées dans les années 1990. Deuxièmement, plusieurs travaux qui ont étudié la relation entre la privatisation et la performance ont conclu à l'amélioration significative de leur ratio d'investissement, d'efficacité, de productivité et de la rentabilité (Nash et al., 1994 ; Newberry et Pollitt, 1997 ; Boubakri et Cosset, 1998, etc.).

Le tableau 1 ci-dessous résume toutes les variables utilisées dans cette étude. 
Tableau 1.Mesures des variables incluses dans l'analyse

\begin{tabular}{|c|c|c|}
\hline Variables & Abréviations & Mesures \\
\hline \multicolumn{3}{|c|}{ Dépendantes } \\
\hline Valeur partenariale & VPA & Ln (valeur ajoutée) \\
\hline Part de valeur & PVPAS & charge de personnel \\
\hline $\begin{array}{l}\text { partenariale allouée } \\
\text { aux salariés }\end{array}$ & & valeur ajoutée \\
\hline \multicolumn{3}{|c|}{ Indépendantes } \\
\hline \multirow[t]{2}{*}{ Actionnariat salarié } & PKSAL & $\begin{array}{c}\text { Pourcentage du capital détenu par les } \\
\text { salariés }\end{array}$ \\
\hline & PSAK & $\begin{array}{c}\text { Variables binaires portant sur la } \\
\text { présence (1) ou l'absence (0) des } \\
\text { salariés au capital }\end{array}$ \\
\hline \multicolumn{3}{|c|}{ Contrôles } \\
\hline Politique & INV & immobilisations corporelles \\
\hline d'investissement & & chiffres d'affaires $H T$ \\
\hline Marge des firmes & $\mathrm{MBE}$ & excédent brut d'exploitation \\
\hline Niveau d'activité & CROIS & $\begin{array}{c}\frac{\text { chiffres d'affaires HT }}{\text { Croissance du chiffre d'affaires HT (2008- }} \\
\text { 2013) }\end{array}$ \\
\hline Taille & TAIL & Logarithme du total des actifs \\
\hline Secteur d'activité & SEC & $\begin{array}{l}\text { Variables binaires }(1: \text { industrie } ; 0 \text { : } \\
\text { autres) }\end{array}$ \\
\hline Temps & TPS & $\begin{array}{c}\text { Nombre d'années l'entreprise dispose } \\
\text { l'actionnariat salarié }\end{array}$ \\
\hline Privatisation & PRIV & $\begin{array}{l}\text { Variables binaires }(1: \text { entreprises } \\
\text { privatisées ; } 0: \text { Non privatisées) }\end{array}$ \\
\hline
\end{tabular}

S'agissant de l'analyse des données, elle a porté sur 31 entreprises à actionnariat salarié sur six années (2008-2013). A cette fin, des régressions linéaires en en données de panel, corrigées pour toute hétéroscédasticité latente et autocorrélation des erreurs, ont été utilisées. En conséquence, ces modèles estimés portent sur 186 observations. Les formes générales de ces régressions sont les suivantes :

$\mathrm{VPA}_{i t}=c+\alpha$ PKSAL $_{i t}+\beta_{1} \mathrm{INV}_{\text {it }}+\beta_{2} \mathrm{MBE}_{i t}+B_{3} \mathrm{CROIS}_{\text {it }}+\beta_{4}$ TAIL $_{i t}+\beta_{5}$ $\mathrm{SEC}_{\text {it }}+\beta_{6} \mathrm{AAS}_{\text {it }}+\beta_{7} \mathrm{PRIV}_{\text {it }}+\varepsilon_{\text {it }}\left(\mathrm{M}_{1}\right)$

PVPAS $_{i t}=c+\alpha$ PKSAL $_{i t}+\beta_{1}$ INV $_{\text {it }}+\beta_{2}$ MBE $_{i t}+\beta_{3}$ CROIS $_{i t}+\beta_{4}$ TAIL $_{i t}$ $+\beta_{5} \mathrm{SEC}_{\text {it }}+\boldsymbol{B}_{6} \mathrm{AAS}_{\mathrm{it}}+\boldsymbol{B}_{7} \mathrm{PRIV}_{\mathrm{it}}+\boldsymbol{\varepsilon}_{\mathrm{it}}\left(\mathrm{M}_{2}\right)$.

L'analyse de ces modèles nécessite le choix des différents tests empiriques. Il s'agit des tests de présence d'effets individuels afin de vérifier l'existence des effets fixes dans les modèles, de Hausman pour effectuer le choix entre le modèle fixe ou le modèle à effets aléatoires, de Breusch Pagan pour vérifier l'hétéroscédascité des résidus et de Breusch Godfrey pour examiner les problèmes d'autocorrélation des erreurs . 


\section{Résultats :}

Dans cette partie, il est question d'exposer les résultats des caractéristiques de l'échantillon, du niveau de participation des salariés au capital, du niveau de création de valeur partenariale, de l'impact de l'actionnariat salarié sur la création de valeur partenariale

Caractéristiques de l'échantillon

L'échantillon de cette étude comporte 89 entreprises réparties en deux catégories :

$>$ Les entreprises à actionnariat salarié (31 entreprises)

$>$ Les entreprises conventionnelles (58 entreprises)

Du sous-échantillon des entreprises à actionnariat salarié, il ressort que la majorité applique cette politique depuis au moins 10 ans. Elles sont toutes des Sociétés Anonymes (S.A) et appartiennent majoritairement au secteur industriel $(45,2 \%)$, puis aux secteurs des services $(22,6 \%)$, de l'agriculture $(16,1 \%)$, du transport $(9,7 \%)$ et du commerce $(6,5 \%)$. Selon le critère des effectifs d'employés, ces entreprises sont en majorité de grande taille ; c'est à dire employant plus de 100 salariés (83,9\% de l'échantillon), seulement 16,1 $\%$ emploient entre 20 et 100 salariés (Moyennes entreprises).

Pour ce qui est des entreprises conventionnelles, la quasi-totalité de ces entreprises sont des grandes tailles, employant plus de 100 employés (93.1\%). La moitié de celles-ci, appartiennent au secteur industriel, tandis que $35 \%$ de ces dernières exercent dans le service et $15 \%$ font dans le commerce. Le tableau 2 ci-dessous récapitule les principaux résultats.

Tableau 2. Répartition de l'échantillon

\begin{tabular}{|c|c|c|c|c|}
\hline & \multicolumn{2}{|c|}{ Entreprises à actionnariat salarié } & \multicolumn{2}{|c|}{$\begin{array}{c}\text { Entreprises } \\
\text { conventionnelles }\end{array}$} \\
\hline & Nombre & Fréquence $(\%)$ & Nombre & $\begin{array}{l}\text { Fréquence } \\
(\%)\end{array}$ \\
\hline \multicolumn{5}{|c|}{ Secteur d'activité } \\
\hline Industrie & 14 & 45,2 & 29 & 50 \\
\hline Service & 07 & 22,6 & 20 & 35 \\
\hline Agriculture & 05 & 16,1 & 0 & 0 \\
\hline Transport & 03 & 9,7 & 0 & 0 \\
\hline Commerce & 02 & 6,4 & 09 & 15 \\
\hline Total & 31 & 100 & 58 & 100 \\
\hline \multicolumn{5}{|c|}{ Ancienneté dans l'actionnariat salarié } \\
\hline 5 à 10 ans & 05 & 16,1 & & \\
\hline 10 ans et Plus & 26 & 83,9 & & \\
\hline Total & 31 & 100 & & \\
\hline \multicolumn{5}{|c|}{ Effectif du Personnel } \\
\hline 20 à 100 & 05 & 16,1 & 04 & 06,9 \\
\hline 100 et Plus & 26 & 83,9 & 54 & 93,1 \\
\hline Total & 31 & 100 & 58 & 100 \\
\hline
\end{tabular}


L'examen du tableau 2 ci-dessous laisse apparaître deux conclusions. D'une part, L'ancienneté dans l'actionnariat salarié est une réalité dans les entreprises de l'échantillon, en ce sens que dans la quasi-totalité de celles-ci $(83,9 \%)$, cette politique existe depuis plus de 10 ans. Ce résultat s'interprète par le fait que l'échantillon est dominé par les entreprises privatisées dans les années 90 dont l'ouverture du capital aux salariés est l'une des conditions nécessaires pour cette privatisation. D'autre part, l'actionnariat salarié est plus pratiqué dans les grandes entreprises (83,9 \%) que dans les moyennes entreprises $(16,1 \%)$. Ce résultat se trouve être en conformité avec les recherches d'Audard et Bachelard (2009) ainsi que celle de Guery (2015) qui prouve que la pratique de l'actionnariat salarié est plus présente dans les grandes entreprises que dans les petites et moyennes entreprises. Ainsi, après présentation des caractéristiques générales des entreprises de l'échantillon, la partie qui suit examine le niveau de participation des salariés au capital.

Le tableau 3 ci-dessous quant à lui, présente le degré d'ouverture du capital aux salariés dans les entreprises au Cameroun.

Tableau 3. Niveau de participation des salariés dans le capital de leur entreprise

\begin{tabular}{crr}
\hline Eléments & Effectifs & Fréquences (\%) \\
\hline Moins de $5 \%$ & 7 & 22,6 \\
Entre $5 \%$ et $10 \%$ & 19 & 61,3 \\
$10 \%$ et plus & 5 & 16,1 \\
\hline TOTAL & $\mathbf{3 1}$ & $\mathbf{1 0 0}$ \\
\hline
\end{tabular}

Les résultats de ce tableau montrent que, dans 61,3\% des entreprises de l'échantillon, le niveau de participation des salariés dans le capital se situe entre $5 \%$ et $10 \%$. Toutefois dans $22,6 \%$ de ces entreprises ce niveau se trouve en dessous de $5 \%$ et dans $16,1 \%$ il se place au-dessus de $10 \%$. Par ailleurs, le niveau moyen de cette participation est à hauteur de $7,4 \%$ du capital. Ce résultat a évolué par rapport à celui de Ngongang (2013) qui trouve un niveau moyen de 2,9\%. Ainsi, ce résultat peut s'expliquer par le fait que, la plus part des entreprises privatisées ont rétrocédé la part de $5 \%$ des salariés prévu par l'ordonnance n $^{\circ}$ 90/004 du 22 Juin 1990 et aussi par la vague de mise en place des plans d'actionnariat salarié dit «Global » observée en 2013, comme le «Share Plan 2013 d'AXA-Assurance et de SANOFI».

La conclusion que l'on peut tirer de ces résultats est que le niveau de participation des salariés au capital est significatif au regard de la moyenne de 7,4 \% de participation. Ce résultat est en conformité avec les standards internationaux en matière de développement d'un «actionnariat salarié significatifs » qui précisent que les entreprises doivent ouvrir le capital à leur employé à hauteur de 3\% (Mathieu, 2016).

Le tableau suivant donne les statistiques descriptives de la création de valeur partenariale dans les entreprises au Cameroun. 
Tableau 4. Distribution moyenne de la création de valeur partenariale dans les entreprises au Cameroun

\begin{tabular}{|c|c|c|c|c|}
\hline variables & \multicolumn{2}{|c|}{ Valeur partenariale (VPA) } & \multicolumn{2}{|c|}{$\begin{array}{l}\text { Part de valeur partenariale } \\
\text { allouée aux salariés(PVPAS) }\end{array}$} \\
\hline Groupes & 1 & $\mathbf{0}$ & 1 & $\mathbf{0}$ \\
\hline Moyenne & 5,04 milliard & 650 million & 0,50 & 0.25 \\
\hline t-test & \multicolumn{4}{|c|}{$14,44 * * *$} \\
\hline Ecart-type & 4.04 & 5,5 & 0,20 & 0,27 \\
\hline $\begin{array}{c}\text { Variance } \\
\text { ratio test }(F)\end{array}$ & \multicolumn{4}{|c|}{$1,5 * * *$} \\
\hline $\begin{array}{c}\text { Nombre } \\
\text { d'observation } \\
\end{array}$ & \multicolumn{4}{|c|}{534} \\
\hline Significativité & \multicolumn{4}{|c|}{${ }^{*} \mathbf{p}<10 \% ; * * \mathbf{p}<5 \%, * * * p<1 \%$} \\
\hline
\end{tabular}

Groupe 1 : entreprises avec actionnariat salarié

Groupe 0: entreprises conventionnelles

Moyenne échantillon : VPA= 1,3 milliard FCFA ; PVPAS = 0,34

Les résultats du tableau 4 montrent que le niveau moyen de création de valeur partenariale dans les entreprises au Cameroun s'élève à 1,3 milliard de francs CFA avec en moyenne $34 \%$ de cette valeur distribuée aux salariés. Toutefois, l'on observe que ce niveau est cinq fois plus élevé dans les entreprises à actionnariat salarié, qui enregistrent une moyenne de 5,04 milliard de francs CFA avec $50 \%$ de cette valeur allouée aux salariés contre 650 million de valeur partenariale créée et seulement $25 \%$ de cette valeur distribuée aux salariés dans les entreprises conventionnelles.

Ainsi, le test de différence de moyenne réalisé sur ces deux groupes d'entreprises confirme que la création et la distribution de valeur partenariale aux salariés dans les entreprises conventionnelles sont inférieures à celles des entreprises à actionnariat salarié. Par ailleurs, d'après les résultats de ce tableau, l'on peut conclure que les variances du niveau de création et de distribution de valeur partenariale aux salariés sont plus élevées dans les entreprises conventionnelles (5,5 contre 4,04 pour la création de valeur partenariale, et 0,27 contre 0,20 pour la distribution de cette valeur aux salariés) que dans les entreprises à actionnariat salarié. Ainsi donc, sur la base du niveau de création et de distribution de valeur partenariale aux salariés le groupe d'entreprises à actionnariat salarié est plus homogène.

Pour ce qui est de l'impact de l'actionnariat salarié sur la création de valeur partenariale, 'examen de matrice de corrélation des variables indépendantes montre que tous les coefficients de corrélation de Pearson sont inférieurs à 0,7 limite à partir de laquelle l'on commence généralement à avoir un problème sérieux de multi colinéarité (Kervin, 1992 Cela indique alors l'absence de multi colinéarité entre les variables indépendantes incluses dans les modèles. S'agissant du test de spécification des effets pour les modèles, le tableau 5 suivant, synthétise les principaux résultats. 
Tableau 5. Synthèse des résultats du test de spécification des effets

\begin{tabular}{|c|c|c|}
\hline \multicolumn{3}{|c|}{ Test de présence des effets individuels } \\
\hline & M1 & M2 \\
\hline Probabilité de Fisher & $4,81 * * *$ & $11,73 * * *$ \\
\hline Significativité & 0.0002 & 0,000 \\
\hline \multicolumn{3}{|c|}{ Test de Hausman } \\
\hline Chi 2 & $34,32 * * *$ & 8,59 \\
\hline Significativité & 0,000 & 0,198 \\
\hline \multicolumn{3}{|c|}{ Test de Breusch Pagan-Heteroskedasticity } \\
\hline Wald chi2(4) & 0,681 & $14,27 * * *$ \\
\hline Significativité & 0,576 & 0,000 \\
\hline \multicolumn{3}{|c|}{ Test de Breusch Godfrey-Auto corrélation des erreurs } \\
\hline Chi 2(1) & $15,345^{* *}$ & 40,727 \\
\hline Significativité & 0,032 & 0,000 \\
\hline
\end{tabular}

L'examen du tableau 5 révèle que les modèles mis en œuvre dans cette recherche sont des modèles à effets spécifiques. De même, le premier modèle $\left(\mathrm{M}_{1}\right)$ est un modèle à effets fixes tandis que le second $\left(\mathrm{M}_{2}\right)$ est un modèle à effets aléatoires.

Pour ce qui est des problèmes d'hétéroscédasticité latente et d'auto corrélation des erreurs, les résultats des tests de Breusch-Pagan et de BreuschGodfrey sont significatifs dans le modèle 2. Par contre dans le modèle 1 , le problème d'hétéroscédasticité ne se pose pas dans la meure le test de BreuschPagan n'est pas significatif. Il se pose plutôt le problème de d'autocorrélation des erreurs dans ce modèle (2).Afin de corriger ces problèmes, la méthode des moindres carrés généralisés est adoptée pour estimer ces modèles. Le tableau 6 ci-dessous expose les principaux résultats.

Tableau 6. Résultats des estimations des modèles

\begin{tabular}{ccc}
\hline \multicolumn{3}{c}{ Variables indépendantes } \\
\hline & Modèle 1 : VPA & Modèle 2 : PVPAS \\
\hline Pourcentage du capital détenu par les salariés & $\mathbf{1 , 6 7 *}$ & $\mathbf{1 , 2 8} * * *$ \\
& $(\mathbf{1 , 8 4})$ & $\mathbf{( 4 , 7 8 )}$ \\
Politique d'investissement & $\mathbf{- 0 , 3 0 * * *}$ & $\mathbf{0 . 0 6} * * *$ \\
& $(-\mathbf{7 , 9 0 )}$ & $\mathbf{( 5 , 0 2 )}$ \\
Marge des firmes & $\mathbf{1 , 3 3} * *$ & $\mathbf{- 1 . 1 2} * * *$ \\
& $(\mathbf{2 , 9 5 )}$ & $\mathbf{( - 8 , 4 2 )}$ \\
Niveau d'activité & 0,15 & $-0,002$ \\
& $(0,69)$ & $(-0,02)$ \\
taille & $\mathbf{0 , 8 9} * * *$ & 0.0009 \\
& $(\mathbf{2 7 , 9 4 )}$ & $(0,10)$ \\
Secteur d'activité & $-0,43$ & $\mathbf{0 , 1 7} * *$ \\
& $(-1,44)$ & $\mathbf{( 2 , 0 3 )}$ \\
Privatisation & $-0,14$ & $\mathbf{- 0 , 3 3} * * *$ \\
& $(-0.65)$ & $\mathbf{( - 4 , 9 9 )}$ \\
Ancienneté dans l'actionnariat salarié & $\mathbf{0 . 0 1} * *$ & $\mathbf{- 0 , 0 0 4} * *$ \\
& $(\mathbf{2 . 2 2})$ & $\mathbf{( - 2 , 3 7 )}$
\end{tabular}




\begin{tabular}{ccc} 
Constance & $1.20 * * *$ & $0,89 * * *$ \\
& $(2.27)$ & $(4,39)$ \\
\hline Log likelihood & $-146,87$ & 80,34 \\
Wald chi2 $(8)$ & 1014,84 & 129,07 \\
Prob $>$ chi2 & 0.000 & 0.000 \\
\hline
\end{tabular}

Statistique $\mathrm{Z}$ entre parenthèse

$* p<0.1 ; p<0.05, * * * p<0.01$

L'examen du tableau 6 révèle que les modèles mis en œuvre dans cette recherche sont de bonne qualité ( $\log$ likelihood $=-146,87$ et 80,$34 ;$ prob $>$ chi2 $=0,000$ ). Par ailleurs, ces résultats montrent, d'une part que la participation des salariés au capital a un impact positif et significatif sur la création de valeur partenariale au seuil statistique toléré de $10 \%$ et d'autre part, elle influence positivement et significativement la part de valeur partenariale allouée aux salariés au seuil statistique de $1 \%$. A la lumière de ces résultats, les hypothèses postulées ci-haut sont confirmées.

Pour ce qui est des variables du contrôle, la marge des firmes, la taille et l'ancienneté dans l'actionnariat salarié sont conformes au sens escompté dans le modèle 1 respectivement au seuil statistique de 5\%, et de $1 \%$. Tandis que la politique d'investissement affecte négativement la valeur partenariale. En outre pour le modèle $2\left(\mathrm{M}_{2}\right)$, la politique d'investissement et le secteur d'activité affectent positivement la valeur partenariale distribuée aux salariés au seuil respectif de $1 \%$ et $5 \%$. Par contre, la marge des firmes, la privatisation et l'ancienneté dans l'actionnariat salarié influencent négativement la valeur partenariale distribuée aux salariés aux seuils statistiques tolérés de $1 \%$ et de $5 \%$.

\section{Discussion}

L'impact positif et significatif de l'actionnariat salarié sur la valeur partenariale peut s'interpréter par le positionnement des salariés actionnaires dans la nouvelle approche de la gouvernance d'entreprise. En effet, dans cette approche, la relation d'agence entre salarié actionnaire et dirigeant est différente de l'approche classique (relation salarié-dirigeant) dans laquelle on peut voir les salariés loués leur capital humain spécifique (Blair, 1997) aux dirigeants et perçoivent en contrepartie une quasi-rente (Rottenberg, 1962). Le schéma conceptuel traditionnel est donc inversé. Le salarié actionnaire n'est pas considéré comme l'agent, mais comme le principal de la relation contractuelle qu'il entretient avec la firme. C'est ainsi que, la détention d'actions par le salarié lui confère certains droits de contrôle qu'il peut exercer en tant que principal dans sa relation d'agence sur le dirigeant, agent de la même relation. Un tel contrôle protège les investissements en capital humain spécifique et par conséquent, encourage leur développement. 
De surcroit, cette détention permet l'alignement des intérêts des salariés actionnaires et des actionnaires externes, ce qui peut favoriser l'instauration d'un régime de confiance dans l'organisation (Charreaux, 1998 ; Poulain-Rehm, 2007) et le développement d'une coopération entre les parties prenantes (Aoki, 1984 ; Marens et Alii, 1999). De telles conséquences contribuent à la réalisation de l'objectif de la firme dans sa conception partenariale, à savoir la maximisation de la valeur partenariale tout en assurant l'équilibre entre les différentes parties prenantes (Fama, 1980 ; Hirigoyen, 1997).C'est dans ce cadre que Poulain-Rehm (2007) précise qu'une politique d'actionnariat salarié exprimant la confiance des dirigeants et des salariés dans l'avenir de leur firme, peut également susciter un sentiment de confiance chez les partenaires financiers ou les fournisseurs de l'entreprise, et ainsi contribuer à la création de valeur partenariale. De même ce mécanisme d'incitation encourage les salariés actionnaires à contrôler aussi bien leurs efforts que ceux des autres salariés. En d'autres termes, l'actionnariat salarié crée une culture de responsabilité collective et une relation de travail positive qui implique la création d'une perception d'intérêt communs et buts partagés (Lawler, 1977). Ceci a été signalé également par Long (1980), qui précise que l'actionnariat salarié procure un sentiment d'avoir des buts communs partagés, un sentiment d'appartenance (ou de solidarité) et de loyauté. L'actionnariat salarié serait susceptible d'être à l'origine d'un plus grand engagement de leur part, d'un plus grand dévouement à long terme et d'une réduction de conflit au sein de l'organisation. Desbrières (2002) ajoute dans ce cadre que : «La pratique de l'actionnariat salarié apparaît être un facteur clé d'intégration des salariés dans l'organisation et l'accroissement des performances individuelles et collectives ». De même, pour Sahut et Gharbi (2012) l'actionnariat salarié est un mécanisme permettant la création de valeur partenariale, favorisant l'engagement et la coopération avec les autres actionnaires, augmentant l'identification et le sentiment d'appartenance à l'entreprise.

En ce qui concerne les variables de contrôle, conformément aux prédictions de Copeland et al. (1991) et de Rappaport (1986), la marge brute des firmes (MBE) reste toujours un facteur de la création de valeur. La taille quant à elle reste dans son rôle de régulateur de fluctuations extérieures. Pour finir la compréhension du mécanisme de l'actionnariat salarié avec les changements au niveau de leur attitude favorise la création de valeur partenariale. Ce qui explique l'effet positif de l'ancienneté dans l'actionnariat salarié sur la valeur partenariale.

Pour ce qui est du modèle 2, les explications que l'on pourra apporter se situent d'un côté, au niveau de la création de valeur partenariale. En effet, la problématique de cette valeur soulève immanquablement celle des destinataires de la richesse créée et, par conséquent, de sa répartition. C'est dans ce sens que, Charreaux (2007) pense que, contrairement à l'approche 
traditionnelle de la gouvernance où l'intégralité du résidu revient aux actionnaires, le recours à l'approche partenariale permet aux différentes parties prenantes d'accéder à la valeur partenariale. De l'autre côté, la métamorphose des jeux de pouvoir internes dans l'entreprise due à la mise en place de la politique d'actionnariat salarié, permet aux salariés de défendre et de protéger leurs propres intérêts, ce qui peut favoriser la répartition et la redistribution de la valeur créée à leur profit. En effet, l'actionnariat salarié peut contribuer à renforcer le contrôle de l'équipe dirigeante par la participation des salariés actionnaires aux organes de gestion (Maalej et Triki, 2008). De même, ils peuvent s'allier avec les actionnaires minoritaires afin d'agir contre leur spoliation par les actionnaires majoritaires. Les actionnaires salariés se distinguent par un niveau de risque plus élevé et une aptitude à acquérir les informations au moindre coût ce qui peut rendre leurs actions plus efficaces.

\section{Conclusion}

L'objectif principal de cette recherche était d'examiner l'impact de l'actionnariat salarié sur la création de valeur partenariale. A ce titre, l'étude conclut à un impact positif et significatif au seuil respectif de $10 \%$ et de $1 \%$ de l'actionnariat salarié sur la valeur partenariale, ainsi que sur la part de valeur partenariale distribuée aux salariés.

Sur le plan théorique, cette étude contribue à enrichir la littérature existante en matière de gouvernance d'entreprise. Plus précisément, elle participe à construire la littérature émergente en finance d'entreprise, relative au pilotage des aspects de la politique d'ouverture du capital aux salariés en apportant un éclairage sur cette politique et en mettant en relief les différentes facettes et stratégies de cette ingénierie financière surtout dans un contexte sans marché financier efficient comme le Cameroun.

Du point de vue managérial, les conclusions de cette recherche peuvent servir de canevas de réflexion aux politiques, managers et dirigeants des entreprises. Ils auront à leur chevet, un outil de management stratégique capable d'améliorer le climat social dans les entreprises et aussi, de favoriser la création de richesse pour tous. C'est une formule qui permet de conjuguer les réalités économiques et sociales dans les organisations.

$\mathrm{Au}$ vu des résultats obtenus, les points suivants doivent être appliqués afin d'améliorer la création de valeur partenariale dans les entreprises au Cameroun. Premièrement, les entreprises appliquant la politique d'actionnariat salarié doivent ouvrir davantage et de manière significative le capital à leurs employés afin d'atteindre le seuil de $25 \%$ du personnel comme salarié actionnaire. Deuxièmement, pour les entreprises conventionnelles, l'ouverture significative d'une partie du capital à leurs employés permettrait de bénéficier de l'aspect positif de l'actionnariat salarié en termes d'implication, de motivation, d'incitation au travail et de création de valeur 
pour tous. Troisièmement, le gouvernement doit créer un cadre juridique afin d'encadrer et inciter les entreprises camerounaises à ouvrir le capital à leurs salariés.

Il est important de souligner à la fin de cette étude que, ces résultats sont à prendre avec beaucoup de prudence à cause de la représentativité de l'échantillon liée à la méthode d'échantillonnage (empirique par convenance) et à la taille de l'échantillon (très réduite). Par ailleurs, cette étude s'est faite dans un contexte sans marché financier efficient.

\section{References:}

1. Audard, L.et Bachelard, A.(2009).L'actionnariat des salariés dans les sociétés françaises: une solution d'avenir?, http://www.asfrance.com/publication-asf/PrixASF/Prix-ASF-2009-actionnariat des-salariés-dans-sociétés-française.pdf

2. Ben-Ner, A. et Jones, D.C. (1995).Employee participation, ownership, and productivity, a theoretical frame framework. Industrial Relations. 34(4), pp.532-554.

3. Blasi, J.R., Conte, M. et Kruse, D. L. (1996).Employee stock ownership and corporate performance among public companies. Industrial and Labor Relation Review, 50(1), pp.60-79.

4. Boubakri, N. et Cosset, J. (1998). The Financial and operating performance of newly-privatized firm: evidence of developing countries. The Journal of Finance, 53, pp. 1081-1110.

5. Buchko, A. (1993).The effects of employee ownership on employee attitudes: an integrated causal model and path analysis, 30(4), pp.633657.

6. Canella, A. (1995). Executives and shareholders: a shift in the relationship. Human Resource Management, Spring, pp.166-184.

7. Chang ,S.and Mayers, D. (1992). Managerial vote ownership and shareholder wealth: evidence from employee stock ownership plans. Journal of Financial Economics, 32(1), pp.103-131.

8. Charreaux, G. (1998).Le rôle de confiance dans le système de gouvernance des entreprises. Economies et Sciences, 8(9), pp.47-65.

9. Charreaux, G.(2007).La valeur partenariale: vers une mesure opérationnelle..... Comptabilité-Contrôle-Audit, 13(1), pp.47-65.

10. Charreaux, G. et Desbrières, Ph.(1998). Gouvernance des entreprises : valeur partenariale contre-valeur actionnariale. Finance Contrôle Stratégie, 20(2), pp.57-88.

11. Clarkson, M.(1995). A shareholder framework for analyzing corporate social performance. Academy of Management Review, 20,(1), 92-117.

12. Collat, D. (1988).In employee ownership and corporate performance: 
a comprehensive review of evidence. National Center for Employee Ownership (http: //www.nceo.org), 2002.

13. D’Arcimoles, C.H. et Tréboucq, S. (2003).Une approche du rôle de l'actionnariat salarié dans la performance et le risque des entreprises françaises.Revue de Gestion de Ressources Humaines, n48, pp.2-15.

14. Desbrières, Ph.(2002).Les actionnaires-salariés. Revue Française de Gestion, 28(141), pp.225-281.

15. Fama, E. (1980). Agency problems and the theory of the firm. Journal of Political Economy, 88, pp.288-307.

16. Gamble, J.E., Culpepper, R. et Blubauch, M. (2002). ESOPs and employee attitudes: the importance of empowerment and final value. Personnel Review, 31(1), pp.9-26.

17. Garvey, G.T. et Swan, P.L. (1994).The economics of corporate governance: beyond the marshallin firm. Journal of Corporate Finance, 1, pp.139-174.

18. Gharbi, H. et Lepers, X.(2008). Actionnariat salarié et enracinement des dirigeants : un essai de compréhension. Innovation, 27, pp.121146.

19. Guedri, Z. et Hollandts, X. (2008a).Beyond dichotomy: The culvilinear impact of employee ownership on firm performance. Corporate Governance : An international Review, 16(5), pp.460-464.

20. Hill, C. et Jones, T. (1992).Stakeholder-agency theory. Journal of Management Studies, 29(2), pp.131-154.

21. Hirigoyen, G. (1997).Salariés-actionnaires: le capital sans pouvoir?, in G. Hirigoyen (Coord.), Histoire, Gestion et Organisation, Presses de l'Université des Sciences Sociales de Toulouse, Toulouse.

22. Jones , J.C. et Kato,T. (1995).The productivity effects of employee ownership plans and bonuses: evidence from japanese panel data. American Economic Review, 85, pp.391-415.

23. Klein, K. (1987).Employee stock ownership and employee attitudes: A test of three models. Journal of Applied Psychology, 73(4), pp.319332.

24. Laffont, J.J. et Martimort, D. (1997). The firm as a multicontract organization. Journal of Economics and Management Strategy, 6(2), pp.201-234.

25. Long R.J. (1980). Job attitudes and organization performance under employee ownership. The Academy of Management Journal, 23(4), pp.726-737.

26. Maalej, H. et Triki, M.(2008). Déterminant de la pratique de l'actionnariat salarié dans les entreprises françaises. La Comptabilité, Le contrôle et 1'Audit entre Changement et Stabilité, pp.1-24. 
27. Marens, R.S., Wicks, A.C. and Hubert, V.L. (1999). Cooperating with the disempowered, using ESOPs to forge a stakeholder relationship employee trust in work place participation programs. Business and Society, 38(1), pp.51-82.

28. Nash, C.A., Preston, J.M. and Shires, J.D. (1994). Rail privatization: the practice an analysis of seven case studies. Working Paper, Institute of Transport Studies, University of Leeds, Leeds, UK.

29. Ngongang, D. (2013).Actionnariat salarié comme levier de création de valeur ajoutée et de productivité dans les entreprises camerounaises. Revue Gestion et Organisation, 5(1), pp.1-8.

30. Newbery, D.and Pollitt, M. (1997). The restructuring and Privatization of Britain's CEGB: was it worth it? Journal of Industrial Economics, 45(3), pp.269-303.

31. Poulain-Rehm, T. (2007).L'actionnariat salarié en France, un facteur de création de valeur ?. Banque \& Marché, 88, pp.25-35.

32. Rottenberg, S. (1962).Property in work. Industrial and Labor Relations Review, 15, pp.402-405.

33. Royer, S. , Waterhouse, J., Brown, K. and Festing, M.(2008). Employee voice and strategic competitive advantage in international modern public corporation: an economic perspective. European Management Journal, 26(4), pp.234-246.

34. Sahut, M. et Gharbi, H.O.(2012), Structure d'actionnariat et performance des firmes : cas des entreprises de l'indice SBF 120, Dissertations, Gratuites.com, à partir de http://www.dissertationsgratuites.com/dissertations/structured\%27Actionnariat/391952.html, Consulté en Mars 2016.

35. Stout, L.A.(2007). Why we should stop teaching dodge? ULCA school of Law, research paper, $\mathrm{n}^{\circ} 07-11$.

36. Trébucq, S. (2002). L'actionnariat salarié dans les entreprises familiales du SBF 250: un outil de création de valeur? Revue Finance Contrôle Stratégie, 15(4), pp.107-453.

37. Trewhitt, L. (2000).Employee buyouts and employee involvement: a case study investigation of employee attitudes. Industrial Relations Journal, 31(5), pp.437-453.

38. Wanda, R.(2001).Structure financière et performance des entreprises dans un contexte sans marché financier: le cas du Cameroun. Revue du Financier, site www.cybel.fr, 12/01/2015.

39. Welbourne, T.et Cyr, L.(1999).Using ownership as an incentive. Group and Organization Management, 24(4), pp.438-460.

Ouvrages

40. Aoki, M. (1984).The cooperative game theory of the firm. Oxford University Press. 
41. Autenne A. (2005).Quelle gouvernance des plans d'actionnariat salarié ? Annales de Droit de l'Université Catholique de Louvain.

42. Blair,M.M. (1997). Firm specific human capital and the theory of the firm. Working paper, The Brookings Institutions, Washington, D.C.

43. Blair, M.M. (1995).Ownership and control: rething corporate governance for the twenty-first century. The Brookings Institutions, Washington, D.C.

44. Blair, M.M. (1996). Wealth creation and wealth sharing. Brookings Institutions.

45. Bompoint, P.et Marois, B.(2004). Gouvernance d'entreprise et communication financière. Economica, Paris.

46. Caby J. et Hirigoyen G. (2001). La création de valeur de l'entreprise. 2ème édition, Ed. Ecomica.

47. Charreaux, G. (1997a).Vers une théorie de gouvernement des entreprises. in Charreaux G. (ed)., Le gouvernement des entreprises. Economica, pp.421- 469.

48. Copeland, T., Koller, T. et Murrin, J.(1991). La stratégie de la valeur. Interdictions.

49. Fédération des actionnairess salariés en Europe (2017). Enquête sur le recensement de l'actionnariat salarié. 6th edition. FAS, Paris.

50. Freeman, R.E.(1984), Strategic management: a stakeholder approach. Pitman-Ballingar.

51. GICAM (2006), Environnement de l'investissement privé au Cameroun. Bulletin n³2, pp.1-46.

52. Guery, L.(2015).L'actionnariat salarié en période de crise : performance économique, performance sociale et questions de gouvernance : preuves empiriques françaises et éclairages britanniques. Recherche effectuée dans le cadre d'une convention conclue entre l'Institut de Recherches Economiques et Sociales (IRES) et la CFE-CGC.

53. Kervin, J. B. (1992).Methods for business research. New-York: Haper Collins.

54. Lawler, E.E.(1977). Reward systems. In J.R.Hackman \&J.L.Suttle(Eds), Improving life at work. Santa Monica, CA: Goodyear.

55. Mathieu, M.(2016).Recensement économique annuel de l'actionnariat salarié dans les pays européens, Fédération Européenne de 1'Actionnariat Salarié, Bruxelle.

56. Pendleton, A.(2001). Employee ownership, participation and governance: a study of ESOPS in UK. London, New-York: Routledge.

57. Rappaport, A. (1986). Creating shareholder value: the new standards of business performance. Free Press. 
Thèses

58. Caramelli, M. (2006). Une étude de l'actionnariat des salariés dans le contexte de l'entreprise multinationale : une approche attitudinale interculturelle. Thèse de Doctorat en Sciences de Gestion, Université de Montpellier II.

59. Dondi, J. (1992).Contribution à la connaissance de l'actionnariat des salariés. Thèse de Doctorat en Sciences de Gestion, Université Bordeaux I.

60. Garfatta, R. (2010). Actionnariat salarié et création de valeur dans le cadre d'une gouvernance actionnariale et partenariale: Application au contexte français. Thèse de Doctorat, IAE, Université de Bourgogne, http://tel.archivesouvertes.

fr/docs/00/59/28/PDF/these_A_GARFATTA_-_Riath_2010.pdf. 